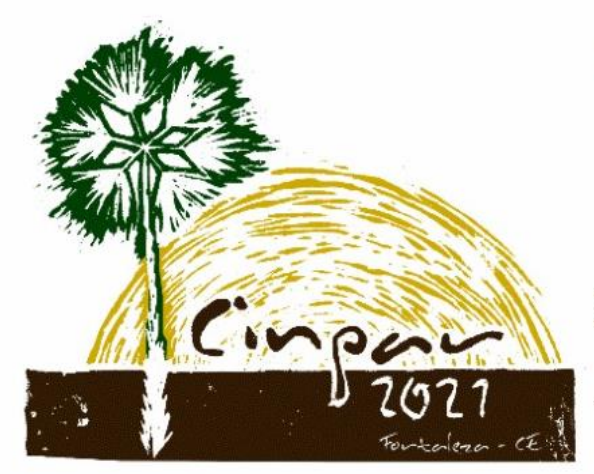

XVII Congresso Internacional sobre Patologia e Reabilitação das Construções

XVII Congreso Internacional sobre Patología y Rehabilitación de las Construcciones

XVII International Conference on Pathology and Constructions Rehabilitation

FORTALEZA (Brasil), 3 a 5 de junho de 2021

https://doi.org/10.4322/CINPAR.2021.131

\title{
Degradações e Vida Útil em Fachadas com Revestimento Cerâmico
}

\section{Degradations and Service Life in Façades with Ceramic Tile Coating}

\author{
Arthur HARLLES ${ }^{1}$, João P. FERNANDES ${ }^{2}$, Lucas R. BEZERRA ${ }^{3}$, Matheus L. NASCIMENTO ${ }^{4}$, Rafaella L. ARAUJO ${ }^{5}$, \\ Sarah C. MARTINS ${ }^{6}$, Virgínia L. FERNANDES ${ }^{7}$
}

\footnotetext{
${ }^{1}$ Centro Universitário Euro-Americano (UNIEURO), Brasília/DF, Brasil, arthurharlles@gmail.com

${ }^{2}$ Centro Universitário Euro-Americano (UNIEURO), Brasília/DF, Brasil, joaopaulofs31@gmail.com

${ }^{3}$ Centro Universitário Euro-Americano (UNIEURO), Brasília/DF, Brasil, lucasrocha0@live.com

${ }^{4}$ Centro Universitário Euro-Americano (UNIEURO), Brasília/DF, Brasil, leonimatheus@gmail.com

${ }^{5}$ Centro Universitário Euro-Americano (UNIEURO), Brasília/DF, Brasil, rafaella.lins@hotmail.com

${ }^{6}$ Centro Universitário Euro-Americano (UNIEURO), Brasília/DF, Brasil, sarahcm2016@gmail.com

${ }^{7}$ Centro Universitário Euro-Americano (UNIEURO), Brasília/DF, Brasil, vivifernandes1207@hotmail.com
}

Resumo: Quando os edifícios falham no desempenho exigido, eles dão origem a patologias que podem ser originadas pela falta de experiência ou desconhecimento sobre o assunto. Devido ao conjunto de fatores, podem ocorrer descolamentos, fissuras, eflorescências, deterioração das juntas, falha de vedação, entre outros nos revestimentos cerâmicos. Nesse sentido, o presente trabalho visa à quantificação dos percentuais totais e de cada patologia em relação à área total de cada fachada de edifícios localizados em Brasília, Brasil. Na metodologia proposta por Silva (2014), são mapeadas as áreas de ocorrência das patologias, classificando por idade, orientação da fachada, prumada e empena, divisão por região e andar, em seguida, a sobreposição de uma malha para contar as patologias usando o software PHOTO SCAN para ajustar as imagens dos edifícios. Posteriormente, três modelos de cálculos são usados para obtenção das curvas de degradação, gerando uma estimativa de vida útil. Em relação aos resultados, observou-se que as patologias de maior incidência são os descolamentos, aberturas e manchas. Com tudo isso, é possível determinar o grau de urgência que o edifício estudado necessita para providenciar medidas de manutenção, para atender os requisitos determinados por uma norma em relação à vida útil.

Palavras-chave: Patologia, Durabilidade, Vida útil, Degradação, Cerâmica.

\begin{abstract}
When buildings fail the required performance, they give rise to the anomalies that can be originated by the lack of experience or lack of knowledge on the subject. Due to the set of factors, detachments, cracks, efflorescence, deterioration of the joints and failure of sealing, among others in the ceramic tile coatings can occur. In this sense, this paper aims the quantification of the percentages of the total and each pathology in relation to the total area of the façade of a building located in Brasília, Brazil. In the methodology proposed by Silva (2014), it is mapped the areas of occurrence of the pathologies classifying by age, orientation of the façade, shaft and gable, division by region and floor, then the overlap of a mesh for counting the anomalies using the PHOTO SCAN software to adjust the images of the buildings. Subsequently, three models of calculations are used to obtain the degradation curves, acquiring an estimate of the useful life. Regarding the results, it was observed that the anomalies of higher incidence are detachments, openings, and stains. With all this, it is possible to determine the degree of urgency that the studied building needs in providing maintenance measures in order to attend the requirements determined by a standard in relation to service life.
\end{abstract}

Keyword: Pathology, Durability, Service life, Degradation, Ceramic 


\section{Introdução}

Os cuidados a serem tomados com as edificações não acabam com o fim de suas construções, pois as mesmas estão sujeitas a vários agentes que influenciam diretamente na existência de manifestações patológicas. Em especial as fachadas por receberem ações diretas do clima, devem receber uma atenção mais especial em relação à manutenção.

Tendo em vista uma melhor compreensão das principais manifestações patológicas presentes em fachadas, foram escolhidos edifícios localizados no Lúcio Costa e Guará 1, Brasília-DF, Brasil, nos quais apresentam alguns destes problemas patológicos. Para que o estudo não agregasse somente informações teóricas, foi realizado também investigação em campo.

Em um edifício podem existir várias manifestações patológicas, porém neste trabalho foram analisadas as que mais ocorrem em fachadas com revestimento cerâmico, sendo elas: descolamento, abertura e eflorescência (mancha).

Sendo assim, o presente artigo tem como objetivo estudar as principais manifestações patológicas apresentadas nas fachadas de revestimento cerâmico, e realizar a estimativa da vida útil através dos percentuais totais de cada patologia em relação à área total da fachada.

\section{Agentes Causadores}

Há variadas razões quando se trata de manifestações patológicas, isso ocorre quando o edifício ou suas partes perdem a capacidade de apresentar desempenho mínimo pré-estabelecido, tendo origem nas fases de elaboração do projeto e na execução do serviço, que podem ocorrer pela falta de experiência ou falta de conhecimento sobre o assunto.

As fachadas, por estarem mais expostas aos efeitos do meio ambiente e ações climáticas, têm maior probabilidade de apresentar deterioração durante sua vida. Entre os fatores externos com maior influência na deterioração da fachada estão às variações térmicas, ação da chuva e umidade.

As variações térmicas podem ser físicas e químicas nos materiais, gerando fissuras, descolamentos e rupturas. A ação da chuva dirigida depende da orientação em relação à incidência de chuva carregada por um vento pluvial dominante. Dessa maneira ocorre também umidade pela entrada de água em forma de vapor, fluindo através de corpos porosos, por infiltração, condensação, e umidade eventual, podendo resultar em eflorescências, manchas e bolor.

Outras razões para essas degradações estão relacionadas à má execução dos processos construtivos, o seu uso, manutenções, por incompatibilidades físicas e químicas, por cargas excessivas, entre outros.

\section{Metodologia}

Para realização do estudo das manifestações patológicas incidentes nas fachadas com revestimento cerâmico, onde as imagens das fachadas são alinhadas através do programa PhotoScan. Logo após, ocorre o mapeamento das manifestações patológicas com auxílio do programa AutoCad, onde são inseridas as malhas de $0,5 \mathrm{~m} \times 0,5 \mathrm{~m}$ sobre a fachada. Mediante a análise por um modelo de cálculo utilizando o método de Fator de Danos, proposta por Silva (2014), onde é possível chegar à variação desses eventos por meio das áreas que apresentam esses danos em relação à área da amostra.

Esse método fundamenta-se, basicamente, em quatro fases como apresenta o fluxograma (Figura 1) que considera as etapas de: levantamento dos dados, tratamento dos dados, análise preliminar, análise final com proposta de modelo de cálculo de degradação e estimativa de vida útil. 


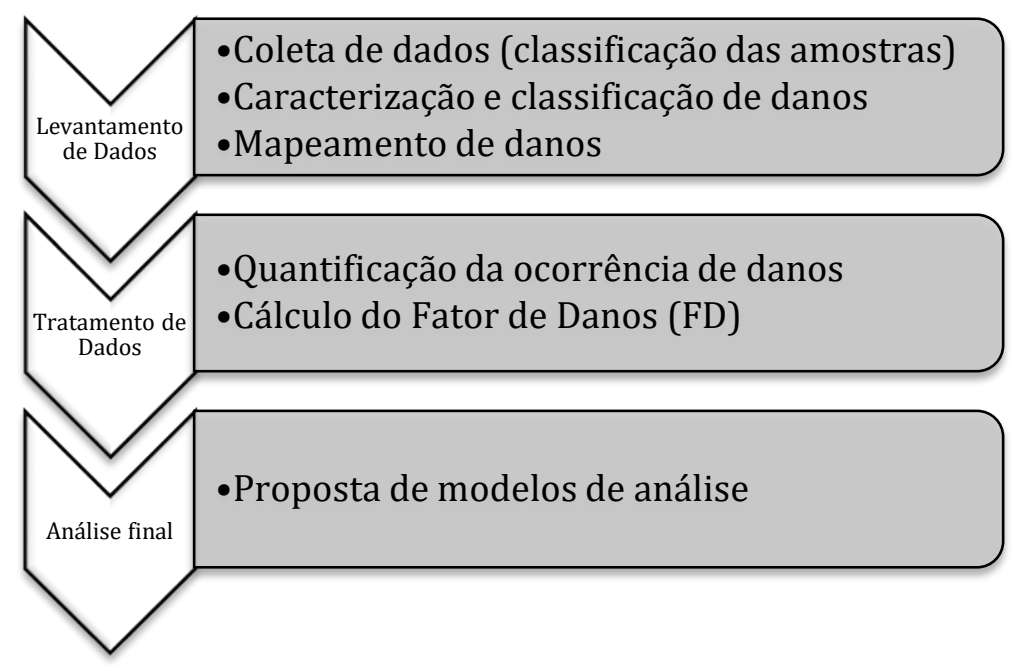

Figura 1 - Fluxograma da metodologia

\section{Análise dos Resultados}

Para uma melhor caracterização das fachadas foi feita uma identificação por prumadas, sendo essas as fachadas de área de maior dimensão do edifício. Para maior compreensão das manifestações patológicas é realizada uma contagem à medida que são identificados em uma malha sobre a amostra. Desta forma as degradações caracterizadas no trabalho foram o descolamento de peças cerâmicas, aberturas e manchas.

Para o mapeamento foi utilizado uma malha sobreposta à amostra (Figura 2), com aberturas de dimensões

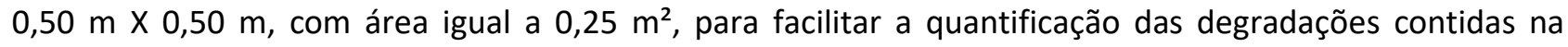
fachada. Cada abertura da malha é uma unidade correspondente a uma patologia, portanto para transformar essas unidades de dano em metro quadrado é necessário multiplicar cada unidade de dano pela área da abertura que corresponde a $0,25 \mathrm{~m}^{2}$. Sendo que esses valores serão os utilizados nos cálculos dos fatores de degradação. (SILVA, 2014).

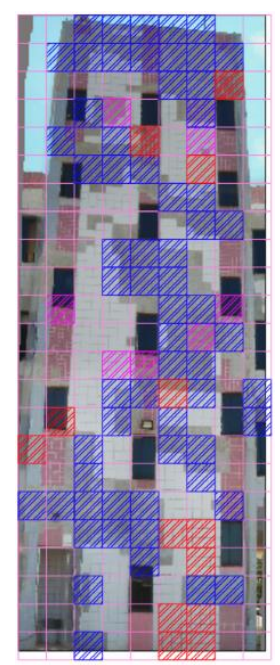

Figura 2 - Mapeamento de danos e Sobreposição de malha nos mapeamentos

\subsection{Cálculo do Fator de Danos}

O fator de danos demonstra através da Equação 1 mostrada abaixo, o primeiro nível do estado de degradação das fachadas, relacionando a área de patologias em função da área total de fachada. Permite definir um parâmetro inicial de estudo de degradação, servindo como base para investigações mais complexas das patologias e consequente estimativa do estado geral de degradação dos sistemas de revestimentos cerâmicos de fachadas (SILVA, 2014). 


$$
F D=\frac{A_{d}}{A} \times 100
$$

Sendo,

FD - Fator de Dano da fachada (\%); Ad - Área de manifestação patológica observada na amostra de fachada $\left(\mathrm{m}^{2}\right)$; A - Área total da amostra de fachada $\left(\mathrm{m}^{2}\right)$.

No presente estudo serão utilizados dois modelos de cálculos de degradação nas fachadas com revestimento cerâmico (SILVA, 2014):

- Modelo baseado no Fator Geral de Danos (FGD);

- Modelo baseado no Fator de Danos Combinado (FDC);

Baseando-se nos 2 modelos de cálculo, será obtida uma representação do avanço da degradação das fachadas.

\subsection{1 - Modelo Baseado no Fator Geral de Danos (FGD)}

A partir da Equação 2 mostrada a seguir, é feita a determinação do valor do FGD das amostras. Desta forma o modelo proposto no presente estudo estabelece uma relação cumulativa ponderada por variáveis (condição da degradação e custo de reparo) condicionantes do processo de degradação (SILVA, 2014).

Onde:

$$
\mathrm{FGD}=\sum\left[\frac{(\mathrm{An} \cdot \mathrm{Kn} \cdot \mathrm{Kc}, \mathrm{n})}{(\mathrm{A} \cdot \mathrm{K})}\right]
$$

FGD - Fator Geral de Danos (\%); An - Área de uma fachada afetada por $n$ tipos de anomalias $\left(\mathrm{m}^{2}\right)$; kn - Nível de condição de $n$ anomalias representando o nível de dano (varia de 0,00 (sem danos) a 4,00 (estado grave de degradação), em escala exponencial); $k$ - Constante, equivalente ao nível da pior condição $(\mathrm{k}=4,00) ; \mathrm{kc}, \mathrm{n}$ - Custo relativo de reparação das anomalias observadas; $\mathrm{A}$ - Área da amostra de fachada $\left(m^{2}\right)$. $n$ - Referência do tipo de anomalia ( $n=1$ - descolamento cerâmico, $n=2$ - aberturas e $n=3$ - mancha).

Para os níveis de condição sua classificação foi feita da seguinte forma, nível 0 - melhor condição, nível 1 - boa condição, nível 2 - degradação suave, nível 3 - degradação elevada e nível 4 - degradação grave (extrema) (SILVA, 2014).

A constante $k$ estabelece o padrão máximo de degradação. Desta forma o estágio mais acentuado ou ainda o referencial máximo da degradação analisada para todos os dados amostrais. Neste estudo em particular, esta constante possui o valor 4,0 , para o prédio 2 ou seja, o maior nível associado aos danos, e para o prédio 1 o valor de 2,00 (SILVA, 2014).

A ponderação dos fatores estabelece a ordem de cada tipo de dano em função da extensão de ocorrência (SILVA, 2014). O Quadro 1 abaixo demonstra os valores dos fatores de ponderação usados para os diferentes tipos de degradação, sendo identificados o edifício 1 como o que se encontra no Lúcio Costa e o 2 no Guará 2.

Quadro 1 - Determinação do estágio de cada dano

\begin{tabular}{|c|c|c|c|c|c|}
\hline \multirow{2}{*}{ Edifício } & $\begin{array}{c}\text { Tipo de dano } \\
\mathbf{( n )}\end{array}$ & $\begin{array}{c}\text { Nível de } \\
\text { condição } \\
\mathbf{( k )}\end{array}$ & $\begin{array}{c}\text { Nível de } \\
\text { ponderação do } \\
\text { dano }(\mathbf{K n})\end{array}$ & $\begin{array}{c}\text { Custo de } \\
\text { reparo } \\
\left(\mathbf{R} \mathbf{S} / \mathbf{m}^{\mathbf{2}} \mathbf{)}\right.\end{array}$ & $\begin{array}{c}\text { Fator relativo de } \\
\text { custo de reparo } \\
\mathbf{( K c , n )}\end{array}$ \\
\hline \multirow{3}{*}{$\mathbf{1}$} & Descolamento & 3 & 2.00 & 113.00 & 1.00 \\
\cline { 2 - 6 } & Aberturas & 0 & 0.00 & 136.70 & 1.21 \\
\cline { 2 - 6 } & Mancha & 1 & 0.02 & 16.00 & 0.14 \\
\hline \multirow{3}{*}{$\mathbf{2}$} & Descolamento & 4 & 4.00 & 113 & 0.83 \\
\cline { 2 - 6 } & Aberturas & 1 & 0.02 & 136.7 & 1.00 \\
\cline { 2 - 6 } & Mancha & 0 & 0.00 & 16 & 0.12 \\
\hline
\end{tabular}


A variável kc,n (Quadro 1) é adimensional e obtida em função da proporção pelo custo de reparo mais elevado, sendo que para o edifício 1 foi utilizado o valor do custo de reparo do descolamento $-\mathrm{R} \$ 113 / \mathrm{m}^{2}$, e para o edifício 2 o custo de reparo de fissura - $R \$ 136,70 / \mathrm{m}^{2}$. Os valores associados à essa variável foram calculados através da composição de custo unitário para serviços de reparos em Brasília (SILVA, 2014).

Para o presente estudo, a partir do método FGD foi obtido os seguintes valores (Quadro 2), sabendo que os cálculos são efetuados para cada amostra e seus respectivos danos.

Quadro 2 - Análise da área de dano

\begin{tabular}{|c|c|c|c|c|c|c|c|}
\hline Edifício & Tipo de dano (n) & As $\left(m^{2}\right)$ & $A\left(m^{2}\right)$ & FD (\%) & Kn & K & $\mathrm{Kc}, \mathrm{n}$ \\
\hline \multirow{3}{*}{1} & Descolamentos & 79.00 & \multirow{3}{*}{210.76} & $37 \%$ & 2.00 & \multirow{3}{*}{2.00} & 1.00 \\
\hline & Aberturas & 0 & & $0 \%$ & 0.00 & & 0.00 \\
\hline & Manchas & 11.50 & & $5 \%$ & 0.02 & & 0.14 \\
\hline \multirow{3}{*}{2} & Descolamentos & 103.50 & \multirow{3}{*}{155.35} & $67 \%$ & 4.00 & \multirow{3}{*}{4.00} & 0.83 \\
\hline & Aberturas & 0.50 & & $0.32 \%$ & 0.02 & & 1.00 \\
\hline & Manchas & 0.00 & & $0 \%$ & 0.00 & & 0.00 \\
\hline
\end{tabular}

Para o presente estudo, a partir do método FGD, utilizando a Equação 2, foi obtido o seguinte valor de degradação total das fachadas para os edifícios 1 e 2 respectivamente.

$$
\begin{gathered}
\mathrm{FGD}^{1}=\left[\frac{(79,00 \cdot 2,00 \cdot 1,00+0,00 \cdot 0,00 \cdot 0,00+11,50 \cdot 0,02 \cdot 0,14)}{210,76 \cdot 2,00} \cdot 100\right] \\
\mathrm{FGD}^{1}=37 \% \\
\mathrm{FGD}^{2}=\left[\frac{(103,50 \cdot 4,00 \cdot 0,83+0,50 \cdot 0,02 \cdot 1,00+0,00 \cdot 0,00 \cdot 0,00)}{155,35 \cdot 4,00} \cdot 100\right] \\
\text { FGD }^{2}=55 \%
\end{gathered}
$$

\subsection{2 - Modelo baseado no Fator de Danos Combinado (FDC)}

O modelo retrata uma interpretação simples do nível de degradação das fachadas, levando em consideração o fator de danos (FD). Este pondera a extensão dos danos diretamente em relação ao custo de reparação das patologias no processo de degradação, utilizando a Equação 3 a seguir (SILVA, 2014).

$$
\mathrm{FDC}=\sum_{1}^{\mathrm{n}}\left(\mathrm{FD}_{\mathrm{n}} \times \mathrm{K}_{\mathrm{c}, \mathrm{n}}\right)
$$

Onde:

$\mathrm{n}$ = tipo de dano (descolamento cerâmico, aberturas e manchas); FD = Fator de Dano; Kc, $\mathrm{n}=$ custo relativo de reparo do dano (Quadro 1);

Com o uso do método FDC, utilizando a Equação 3 , foi encontrado os seguintes valores para os edifícios 1 e 2 respectivamente.

$$
\begin{gathered}
\mathrm{FDC}^{1}=\left[\frac{(79,00 \cdot 1,00+0,00 \cdot 0,00+11,50 \cdot 0,14)}{210,76} \cdot 100\right] \\
\mathrm{FDC}^{1}=38 \% \\
\mathrm{FDC}^{2}=\left[\frac{(103,50 \cdot 0,83+0,50 \cdot 1,00+0,00 \cdot 0,00)}{155,35} \cdot 100\right] \\
\mathrm{FDC}^{2}=56 \%
\end{gathered}
$$




\section{Conclusão}

Efetuado o mapeamento das fachadas dos edifícios por meio da malha sobreposta nas imagens que foram previamente ortogonalizadas no software PHOTO SCAN, pode-se identificar e classificar as manifestações patológicas presentes, com isso foi possível a obtenção da área danificada em relação à extensão total do edifício.

A partir desses dados foi obtido o FD, onde foi viável observar que as manifestações presentes no edifício 1 do Lúcio Costa foram o descolamento das placas cerâmicas com $37 \%$, manchas com $5 \%$ e aberturas com $0 \%$, e para o edifício 2 do Guará I foram descolamento das placas cerâmicas com $67 \%$, aberturas com $0,32 \%$ e manchas $0 \%$, diante disso a maior incidência nos edifícios foi o descolamento das placas cerâmicas. Com o FD que foi aplicado nos modelos para se obter os valores de FGD e FDC, foi identificado o edifício 2 do Guará I com o que tem as maiores porcentagens de manifestações patológicas com FGD de $55 \%$, sendo assim terá um custo maior nos reparos.

A metodologia proposta identifica as patologias e informa uma ordem de quais problemas estão mais avançadas o que pode ajudar na manutenção da fachada, pois poderá determinar qual patologia necessita da manutenção primeiro. Com base no que foi observado pode se concluir que esse método é bastante visual, o que depende da observação do profissional podendo aparentar uma variação nos resultados, se, por exemplo, duas pessoas aplicarem os métodos na mesma fachada.

\section{Referências Bibliográficas}

Goldberg, R. P. Direct adhered ceramic tile, stone, and thin brick facades. Technical Design Manual. Laticrete International, Inc. The USA. 1998.

Barbosa, A. S. Estudo numérico-computacional e analítico do choque térmico em fachadas de edificações. Dissertação de Mestrado, Universidade de Brasília, Brasília, 2013, 275 p

Silva, A. F. Manifestações patológicas em fachadas com revestimentos argamassados: estudo de caso em edifícios em Florianópolis. Florianópolis, 2007, 190 p.

Transforma Engenharia. O que é manutenção predial?. Disponível em: < http://transforma.eng.br/blog-deengenharia/o-que-e-manutencao-predial>. Acesso em: 23 de Maio de 2019.

COBREAP - Congresso Brasileiro de engenharia de avaliações e perícias, 14, Bahia. Congresso Brasileiro de engenharia de avaliações e perícias. Perícia em sistema de revestimento cerâmico de fachadas. 26P.

Rhod,A. Manifestações patológicas em revestimentos cerâmicos: análise da frequência de ocorrência em áreas internas de edifícios em uso em Porto Alegre. Trabalho de diplomação - Universidade federal do Rio Grande do Sul escola de engenharia departamento de engenharia civil, Porto Alegre, 2011. 69P.

Silva, M. N. B. (2014). Avaliação Quantitativa da Degradação e Vida Útil de Revestimentos de Fachada Aplicação ao Caso de Brasília/DF. Tese de Doutorado em Estruturas e Construção Civil, Publicação E.TD006A/14, Departamento de Engenharia Civil e Ambiental, Universidade de Brasília, Brasília, DF, 198 p.

Bauer, E.; Castro, E. K.; Antunes, G. R. e Leal, F. E. Identification and Quantification of Pathologies in Facades of New Buildings in Brasilia, XII DBMC International Conference on Durability of Building Materials and Components, Porto, Portugal, 2011.

Bordalo, R.; Brito, J. P.; Gaspar, L.; Silva, A. Abordagem a um modelo de previsão da vida útil de revestimentos cerâmicos aderentes, Service life prediction modelling of adhesive ceramic tiling systems. Teoria e Prática na Engenharia Civil, Portugal, 2010, n.16, pp. 55-69.

Sousa, R. D. B. Previsão da vida útil dos revestimentos cerâmicos aderentes em fachadas. Dissertação de Mestrado, Instituto Superior Técnico, Universidade Técnica de Lisboa, Portugal, 2008.

Fiber Sals. Juntas de dilatação e os efeitos das variações climáticas nas estruturas. Disponível em: https://fibersals.com.br/blog/juntas-de-dilatacao-e-os-efeitos-das-variacoes-climaticas-nas-estruturas/. Acesso em: 30 de Maio de 2019. 
Construido Decor. Vergas, contravergas e cintas de amarração na alvenaria. Disponível em: http://construindodecor.com.br/vergas-contravergas-cintas-de-amarracao/. Acesso em: 29 de Maio de 2019.

Mapa de Obra. Votorantim soluciona problema de desplacamento de pisos cerâmicos em residência. Disponível em: https://www.mapadaobra.com.br/inovacao/votorantim-soluciona-problema-dedesplacamento-de-pisos-ceramicos-em-residencia/. Acesso em: 29 de Maio de 2019.

IBDA - Fórum da Construção. Cerâmica Soltando: Diagnóstico, Causas e Recuperação. Disponível em: http://www.forumdaconstrucao.com.br/conteudo.php?a=17\&Cod=2034. Acesso em: 30 de Maio de 2019.

TÉCHNE. Patologias cerâmicas. Disponível em: http://techne17.pini.com.br/engenhariacivil/116/artigo287385-3.aspx. Acesso em: 30 de Maio de 2019.

PINI. Qual é o motivo das trincas que aparecem inclinadas nos cantos superiores de portas e janelas e cantos inferiores de janelas?. Disponível em: http://piniweb17.pini.com.br/construcao/noticias/qual-eo-motivo-das-trincas-que-aparecem-inclinadas-nos-83997-1.aspx. Acesso em: 3 de Junho de 2019.

Mapa de Obra. Traço de concreto: a importância do controle de água. Disponível em: https://www.mapadaobra.com.br/inovacao/traco-de-concreto-a-importancia-do-controle-de-agua/. Acesso em: 3 de Junho de 2019.

IAU - USP. Juntas de movimentação. Disponível em: https://www.iau.usp.br/pesquisa/grupos/arqtema/guiaceramicacompleto/02/content/020602_juntas_ movimentacao.htm\#. Acesso em: 3 de Junho de 2019. 